\title{
Fractal based Image Compression Techniques
}

\author{
Sandhya Kadam \\ Research Scholar, Faculty of Engg, Pacific Academy \\ of Higher Education and Research, Udaipur, India
}

\author{
Vijay Rathod \\ St. Xavier's Institute of Technology \\ Mumbai, India
}

\begin{abstract}
Fractal image compression offers high compression ratios and quality image reconstruction. It uses various techniques as the fractal with DCT, wavelet, neural network, genetic algorithms, quantum acceleration etc. Additionally, because fractals are infinitely magnifiable, fractal compression is resolution independent and so a single compressed image can be used efficiently for display in any image resolution including resolution higher than the resolution of the original image. Breaking an image into pieces and identifying selfsimilar ones is the main principle of the approach. In this paper, the different issues in fractal image compression as partitioning, larger encoding time, compression ratio, quality of the reconstructed image, decoding time, SSIM(Structured Similarity Index) are discussed and highlighted. The various areas for improvement as larger encoding time and PSNR are also suggested. The various parameters for evaluating the performance of these techniques as PSNR, compression ratio, encoding time, and decoding time are also suggested. Comparison of Fractal techniques for color image, texture and satellite image is done using different parameters as compression time, compression ratio and PSNR. The hybrid method which combines Fractal quad tree with wavelet and Huffman coding is implemented and compared different parameters as compression ratio and the compression time of the proposed method with the existing methods.
\end{abstract}

\section{General Terms}

Discrete Cosine Transform, Fractal Image Compression, Partitioning Algorithm, Self-Similarity. Wavelet Transform.

\section{Keywords}

Discrete Cosine Transform, Fractal Image Compression, Partitioning, Affine Transformations, PSNR, Quad tree, Self Similarity, Wavelet Transform.

\section{INTRODUCTION}

Fractal image compression is a promising technique for compact storing of images. It is based on fractals and uses self-similarity that can be found in any image. Fractal image compression is a lossy method which is no problem for applications like moving images and also for presenting images on the internet. Standard image compression methods can be evaluated using their compression ratio. It is the ratio of the memory required to store an image as a collection of pixels and the memory required to a representation of the image in compressed form. The compression ratio for the fractal scheme is easy to misunderstand since the image can be decoded at any scale. Monochrome images can be encoded because color images are typically extensions of the gray scale representations of an image.

\section{LITERATURE REVIEW}

The block-based Fractal Image Compression (FIC-B) algorithm by Fisher [1] partitions the image into two basic elements: range blocks and domain blocks. The range blocks form a tiling of the image. The domain blocks are twice the size of the range blocks and overlap such that a new domain block starts at every pixel. Determining the global transformation for the image requires for each range block finding a domain block that can be mapped onto a close approximation of the range block via a predefined set of contractive transformations. These transformations contract the domain blocks spatially to the size of range block and then transform the grey levels by a combination of rescaling, offset adjustment, reflection, and rotation. The rescaling is restricted to a range that ensures a contractive transformation. The method of finding a suitable domain block for a given range block is a search method using a mean squared error distance measure. For each range block in the image, a search of all the transformed domain blocks is made and the distance between the range and transformed domain blocks is calculated using a mean-squared-error distance method. The block with the smallest distance is chosen, and the corresponding transformation is stored.

The global transformation of the image onto a close approximation of itself is then constructed from a combination of these local block transformations. The parameters for these transformations can then be encoded and transmitted or stored. Some form of entropy coding is usually employed. At the decoder, the transformation parameters are reconstructed from the variable length codes. The transformations are then recursively applied to an initial image. Convergence to the fractal image occurs after about 8-10 iterations. The fractal image is an approximation of the encoded image and is dependent only on the transformations. For the standard Lena image ( $512 \times 512$ pixels, 8 bits per pixel), this basic algorithm achieves a compression ratio of 19:1 at a peak signal to noise ratio (PSNR) of $27.9 \mathrm{~dB}$.

Adaptive quad tree method [2] decreases the coding time. Here, an image is divided into four blocks of equal size which are joined by four nodes. The sub-blocks are judged to be sub divided or not depending on statistical properties of the block. In block classification and quad tree partitioning method, subblocks are classified in each level. The range blocks are directly classified while domain blocks are transformed by spatial scaling and spatial position transformations before classification. Thus, searching time is minimized.

Fractal compression [3] is based on the scheme that affine similarity between two image blocks is equivalent to the absolute value of Pearson's correlation coefficient (APCC) between them. In this scheme, all the domain blocks are classified into three classes based on APCC and domain block is searched for range block with closest APCC. Encoding time is significantly reduced with the well preserved image quality of the reconstructed image. 
Adaptive fractal compression method [4] uses Particle Swam Optimization (PSO) for searching. Here, searching time is reduced and hence, encoding time of fractal image compression, but with $1.2 \mathrm{~dB}$ loss in image quality. This can be improved by applying classification techniques for various fractal image compression methods.

Fractal coding is used for compression of medical images [5]. Fractal quasi-lossless and improved quasi-lossless methods are implemented for medical images and results are compared. Both the methods are found to compete with the standard fractal image compression algorithms.

Fast classification based fractal image compression [6] achieves speed up and reach the theoretical time limit of "No search method". Here, the exhausting search process can be reduced by dividing both sets of the domain and the range blocks into subsets with the help of a hashing parameter. By using this classification, one assumes that the hashing parameter values of a given range block and its best matching domain block pair are equal. So the range block and its pair are supposed to be in the same class or subset. The classification is a part of the pre-process executed before the search phase.

An accelerating algorithm based on one norm of the normalized block [7] is based on a special measure called onenorm of a normalized block. Here, a kick out method is used to discard impossible domain blocks in early stage for the current range block. It speeds up efficiently. Further speed can be improved by combining this method with DCT inner product method.

To speed up baseline fractal coding, a new fast encoding method [8] uses a special feature of an image as criteria between two images. This method has much better potential for many applications than baseline fractal coding. Many existing complexity reduction techniques can be incorporated into this method to achieve still better performance. Fast fractal coding [9] uses a special image feature which speeds up the encoding process and can be used to reveal texture and edge information in the images.

To accelerate fractal coding, range and domain blocks are categorized by fuzzy c-mean clustering approach and compared with the use of new metric based on discrete cosine transform coefficient [10]. It is 40 times faster than baseline fractal method and has smaller PSNR decay.

The adaptive wavelet tree based fractal image compression methods [11] with a four-fork tree has a solution to the limitation of consideration of self-similarity. The proposed algorithm clearly increases the compression rate and speed of encoding without reducing SNR and the quality of decoded images as compared to the classical wavelet tree based algorithm for fractal image compression.

In Huber fractal image compression [12], the linear Huber regression technique is embedded into the encoding procedure of the fractal image compression. The main disadvantage of Huber fractal image compression is the high computational cost. To overcome this drawback, particle swam optimization (PSO) technique is utilized to reduce the searching time.

Fast and efficient hybrid fractal-wavelet coder [13] that applies speed of the wavelet transform to the image quality of the fractal compression. Hybrid coder performance is better than that of pure methods. The blockiness in the proposed method is avoided because the fractal stage which uses block partitioned processing only in the approximation sub band, and not in the entire image. Furthermore, the wavelet progressive transmission characteristic is slightly reduced but maintained. Fast hybrid methods would allow extension of fractal Techniques to communicate fast.

Fast hybrid fractal image compression using an image feature and neural network [14] uses a special neural network to modify the mapping scheme for the sub-blocks in which pixel values fluctuate greatly.

In quantum accelerated fractal image compression [15] uses quantum mechanics based on Grover's quantum search algorithm to speed up encoding. The proposed method is more powerful than the other state of the art fractal image compression approaches in maintaining the quality of the retrieved images.

Hybrid fractal image coding with quad tree based progressive structure [16] can be compatible with the pure no search method by adjusting threshold parameter. The proposed scheme outperforms JPEG at a high compression ratio. JPEG2000 gives the best performance among three methods. This method is better than the progressive transmission structure without considering the quad trees.

Genetic algorithm based on discrete wavelet transformation for fractal image compression [17] is implemented to overcome drawbacks of time-consuming fractal coder. The proposed method is 100 times faster than the full search method. Fractal image compression [18] is used in image sharpening and image smoothing. The affine parameters of FIC can vary with different image quality measurements, and they also directly affect the visual quality of a decoded image. Taking advantage of the positive correlation between the decoded image contrast and the parameter ' $\mathrm{s}$ ', an image can be sharpened or smoothed by justifying the values of 's' in the fractal coding system. When's'>SSIM, the decoded image is sharper than the original image. When's' $<$ SSIM, the decoded image is smoother than the original image. When's' = SSIM, the decoded image has best image contrast with the original image.

Fractal compression coding based on wavelet transform with diamond search [19] enhances searching and hence coding speed compared with other image compression techniques under the condition of specific error threshold. But the shortages of this algorithm are blocking artifacts of the decoded image and the decaying of SNR. The further research can be done to improve image quality by solving the problems of this algorithm.

Fractal image compression uses spatial correlation and hybrid genetic algorithm [20] which is based on the characteristics of fractal and partitioned iterated function system. It consists of two stages. The first stage makes use of spatial correlation in images for both range and domain pool and second stage adopt simulating annealing genetic algorithm. To have rapid convergence, the algorithm adopts dyadic mutation operator. It has saved encoding time and obtained high compression ratio.

An automatic region-based video sequence codec based on fractal compression [21] uses intra frame coding. It also uses deblocking loop filter and sub pixel matching to increase the quality of the decoded image. This algorithm is compared with H.264 [22]. It can save compression time with degradation in image quality with respect to H.264. In metaheuristic algorithm [23], diffusion property in random fractals is used. 
The decoding scheme simply consists of iterating the transformations on any initial image until convergence to a final decoded image is observed. The transformation of an image is done sequentially. For each region transformation index $\mathrm{i}$, the transformation $T_{i}$ is applied to the image region over the domain $D_{i}$ and mapped onto the range $R_{i}$.

Different Partitioning schemes [26] are studied. Partitions can be rectangular or triangular. In triangular partitioning, a rectangular image is divided diagonally into two triangles. Each of these triangles is recursively subdivided into four triangles by segmenting the triangle along lines that join three partitioning points along the three sides of the triangle. Quad tree and HV partitioning schemes are also used.

A quad tree partition is a representation of an image as a tree in which each node, corresponding to a square portion of the image contains four sub-nodes, corresponding to the four quadrants of the square. The root of the tree is an initial image. In a quad tree partition, a square in the image is broken up into four equal sized sub-squares. This process repeats recursively starting from the whole image and continuing until the squares are small enough to be covered within some specified RMS tolerance. Small squares can be covered better than large ones because contiguous pixels in an image tend to be highly correlated. A weakness of quad tree based partitioning is that it makes no attempt to select the domain pool in a context dependent way.

In HV partition, a rectangular image is recursively partitioned either horizontally or vertically to form two new rectangles. The partitioning repeats recursively until a covering tolerance is satisfied. This scheme is more flexible since the position of the partition is variable.

Optimization of search procedures and domain pool classification [27, 28] is studied. The domain to range comparison step of the encoding is very computationally intensive. A classification scheme can be used to minimize the number of domains compared with a range.

Vector quantization codebooks can be used for classification of domain blocks. In adaptive Code book clustering, the codebook is to be divided into subsets or clusters. Complexity reduces due to clustering procedure. Cluster size may vary. The computational load can be reduced. The image quality may slightly be affected.

Fractal methods which combine discrete cosine transform (DCT) are studied [29-32]. Fuzzy based methods [33] are also studied. Performance parameters such as encoding time should be minimized [34-35], decoding time, compression ratio, PSNR (Peak Signal to Noise ratio) etc. are analyzed for different fractal coding methods. Encoding time can be significantly reduced by classifying the ranges and domains. Both ranges and domains are classified. Considerable timesaving results using domains in the same class as given range.

Applications of fractal coding to color images [36-38] are also studied. It is fortunate that human visual system uses only three color channels to encode color information that is sent to the brain. This means that colors which correspond to different wavelengths of light can be simulated by a superposition of three primary colors typically, red, green and blue. When a color image is digitized, three filters are used to extract the red, green and blue (RGB) intensities in the image and when these are recombined, we perceive them as some color. A method that can encode a monochrome image can be used to encode color images. Encoding Red, Green and Blue components separately are not reasonable because a human visual system is not practically sensitive to color information. It is possible to significantly compress I and Q signals with little to no apparent degradation. The I and Q channels are typically decimated to one half or one quarter to their original size and compressed usually at lower bit rate than the $\mathrm{Y}$ channel.

Different fractal compression methods are studied [39-42]. Fractal techniques are also used for medical Imaging [43]. Some genetic algorithms [44-45] are also used to improve searching for the domain block. Comparison of basic algorithms [46] is done.

\section{DESIGN OF A FRACTAL IMAGE CODING SYSTEM}

Three main issues in the design and implementation of a fractal block coding system are image partitioning, distortion measure and a class of discrete image transformations.

\subsection{Image Partitioning}

A partition constructed is image dependent. It allows the coder to use larger blocks to take advantage of smoothly varying image blocks. It allows exploitation of redundancy in smooth image areas and leads theoretically high compression ratios. To use small blocks to capture detail in complex areas. Small blocks are easy to analyze and classify geometrically. They allow fast evaluation of inter block distances. They are easy to encode accurately. They lead to robust system whose performance is steady.

\subsection{Distortion Measure}

A distortion measure between digital images is constructed from an inter block distortion measure. The mean squared distortion between the images is defined as the sum over the image, of the squared differences of pixel values.

\subsection{A Class of Discrete Image Transformations}

Determining the global transformation for the image requires for each range block, finding a domain block that can be mapped onto a close approximation of the range block via a predefined set of contractive transformations. The general form of image transformation is a union of a domain to range region transformations. The spatial dimension is a spatial contraction by a factor of two together with $x$ and $y$ translation. The gray level value of each pixel in the contracted image region is the average value of four neighboring pixels in the domain region. The gray level transformations include a combination of rescaling, offset adjustment, reflection and rotation.

\subsubsection{Isometries}

Following is the list of transformations that do not modify pixel values but they simply shuffle pixels within a range block in a deterministic way, we call them Isometries.

Here, $\mu(I, j)=$ range block with indices $i, j$. $\mathrm{B}=$ size of range block 
Table 1. Isometries

\begin{tabular}{|c|c|c|}
\hline $\begin{array}{l}\text { Sr. } \\
\text { No. }\end{array}$ & Isometries & Explaination \\
\hline 1 & $(\mathrm{t} 0 \mu) \mathrm{i}, \mathrm{j}=\mu(\mathrm{i}, \mathrm{j})$ & Identity. \\
\hline 2 & $(\mathrm{t} 1 \mu) \mathrm{i}, \mathrm{j}=\mu(\mathrm{i}, \mathrm{B}-1-\mathrm{j})$ & $\begin{array}{l}\text { Orthogonal } \\
\text { reflection about } \\
\text { mid-vertical } \\
\text { axis } \mathrm{j}=(\mathrm{B}-1) / 2 \\
\text { of the block. }\end{array}$ \\
\hline 3 & $(t 2 \mu) i, j=\mu(B-1-i, j)$ & $\begin{array}{l}\text { Orthogonal } \\
\text { reflection about } \\
\text { mid-vertical } \\
\text { axis } \mathrm{i}=(\mathrm{B}-1) / 2 \\
\text { of the block. }\end{array}$ \\
\hline 4 & $(t 3 \mu) i, j=\mu(j, i)$ & $\begin{array}{l}\text { Orthogonal } \\
\text { reflection about } \\
\text { first diagonal } \mathrm{i} \\
=\mathrm{j} \text { of the block. }\end{array}$ \\
\hline 5 & $\begin{aligned}(t 4 \mu) i, j=\mu(B-1 & -j, B \\
& -1-i)\end{aligned}$ & $\begin{array}{l}\text { Orthogonal } \\
\text { reflection about } \\
\text { second } \\
\text { diagonal } i+j \\
=B-1 \text { of the } \\
\text { block. }\end{array}$ \\
\hline 6 & $(\mathrm{t} 5 \mu) \mathrm{i}, \mathrm{j}=\mu(\mathrm{j}, \mathrm{B}-1-\mathrm{i})$ & $\begin{array}{l}\text { Rotation } \\
\text { around the } \\
\text { center of the } \\
\text { block through } \\
+90 .\end{array}$ \\
\hline 7 & $\begin{aligned}(t 6 \mu) i, j=\mu(B-1 & -i, B \\
& -1-j)\end{aligned}$ & $\begin{array}{l}\text { Rotation } \\
\text { around the } \\
\text { center of the } \\
\text { block through } \\
+180 \text {. }\end{array}$ \\
\hline 8 & $(t 7 \mu) i, j=\mu(B-1-i, j)$ & $\begin{array}{l}\text { Rotation } \\
\text { around the } \\
\text { center of the } \\
\text { block through - } \\
90 .\end{array}$ \\
\hline
\end{tabular}

\subsubsection{Fractal Code}

Mean squared error (MSE) distance method is used as a search method. For each range block, a search of all transformed domain blocks is made. The block with shortest MSE distance is chosen and corresponding transformations are stored. The parameters for this transformation can be encoded as affine maps.
Thus a fractal image code consists of the description of the image partition, gray level offset and one of eight isometries. For efficient storage and to increase compression, bit allocation schemes are used. Three bits are used to store orientation of the domain range mapping.

\section{COMPARISON OF FRACTAL BASED IMAGE COMPRESSION METHODS}

Fractal based techniques are more suitable to give higher compression ratio. Performance comparison of different algorithms in terms of searching time in seconds, compression ratio and PSNR is done for existing transform based methods and analysis is done. These transform based methods are Discrete Cosine Transform based fractal image compression and Quad tree fractal decomposition with Huffman coding. As Fractal image compression methods require more searching time, hybrid method is used for comparison as Quad Tree Fractal decomposion with Huffman coding. Table 2 gives compression ratio, compression time and PSNR for Quad tree fractal decomposition with Huffman Coding. The images are from standard image database.

Table 3 shows results for DCT based Fractal Image Compression. The graphical representation of results of both the existing fractal based techniques is shown in Fig. 1 and Fig. 2 respectively. The comparison is done for compression ratio obtained for Lena, Satellite and Texture images. The compression ratio obtained for Satellite image is higher than than Texture and gray scale image as Lena using Quad Tree Fractal decomposion with Huffman Coding. But DCT based fractal method gives more compression for Lena as Gray scale image as compared to Texture and Satellite image

It is seen that compression ratio (CR) is high for DCT-FIC as compare to QDHC, without degrading quality of the reconstructed image. Though, it is seen that DCT-FIC has larger compression time than the QDHC, it is considered to be more efficient on concerning domain-range based search algorithms.

Hybrid technique is proposed for wavelet based approach. Here, wavelet based Quad tree with Huffman Coding is proposed with wavelets with different levels and obtained experimental results for comparison with the existing methods. Haar wavelet is used in the proposed system.

Table 2. Quad Tree Fractal Decomposition with Huffman Coding

\begin{tabular}{|c|c|c|c|}
\hline $\begin{array}{c}\text { Image } \\
\mathbf{5 1 2 X 5 1 2})\end{array}$ & $\begin{array}{c}\text { Compression } \\
\text { Ratio }\end{array}$ & $\begin{array}{c}\text { Compression } \\
\text { Time (s) }\end{array}$ & PSNR \\
\hline Lena & 10.12 & 1.86 & 25.69 \\
\hline Texture & 17.83 & 1.95 & 28.05 \\
\hline Satellite & 25.65 & 0.78 & 27.74 \\
\hline
\end{tabular}


Table 3. DCT based Fractal Image Compression

\begin{tabular}{|c|c|c|c|}
\hline $\begin{array}{c}\text { Image } \\
\mathbf{( 5 1 2 X 5 1 2 )}\end{array}$ & $\begin{array}{c}\text { Compression } \\
\text { ratio }\end{array}$ & $\begin{array}{r}\text { Compression } \\
\text { Time(s) }\end{array}$ & PSNR \\
\hline Lena & 37.44 & 2.08 & 34.02 \\
\hline Texture & 22.58 & 4.30 & 38.36 \\
\hline Satellite & 27.90 & 3.69 & 34.31 \\
\hline
\end{tabular}

Compression ratio

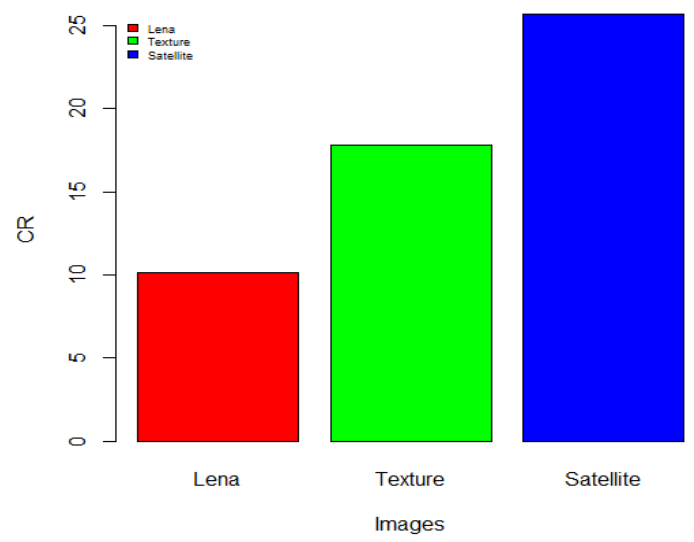

Fig 1: Comparison of compression ratio for Lena, Texture and Satellite Image using Quad tree with Huffman coding

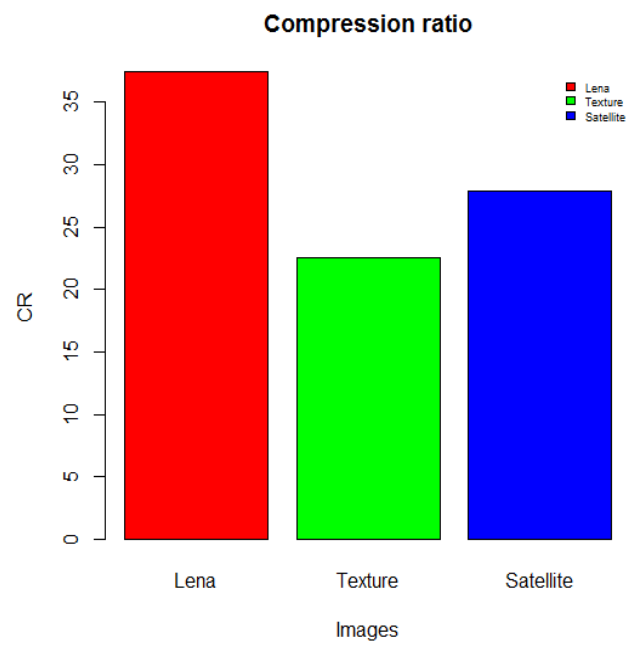

Fig 2: Comparison of compression ratio of Lena, Texture and Satellite image using DCT based fractal coding

\section{PROPOSED SYSTEM}

The proposed system is Discrete Wavelet-based Quad tree with Huffman coding for color images. The discrete Wavelet transform is an efficient tool with some limitations for various image processing applications as in motion estimation, data compression, denoising, and segmentation and classification areas. The discrete wavelet transform is obtained by filtering the signal through a series of digital filters at different scales. The scaling operation is done by changing the resolution of the signal by the process of sub sampling. The DWT can be computed using either convolution based or lifting based procedures. In both methods, the input sequence is decomposed into low pass and high passes sub bands, each consisting of half the number of samples in the original sequence.

The most commonly used wavelets Haar wavelets, Morlet wavelets, Mexical-hat wavelet. The Haar wavelet is discontinuous in time. It is localized in the time domain, but it has poor localization in frequency domain. Discrete wavelet transform using Haar wavelet can be combined with the fractal quad tree with Huffman to improve the compression. Discrete Wavelet-based quad tree combined with Huffman coding is shown in fig 4. The discrete wavelet transform approach is combined to improve the compression ratio and reduce the encoding time as compared to fractal coding method. The lossy and lossless compression methods are combined in this proposed Hybrid system.

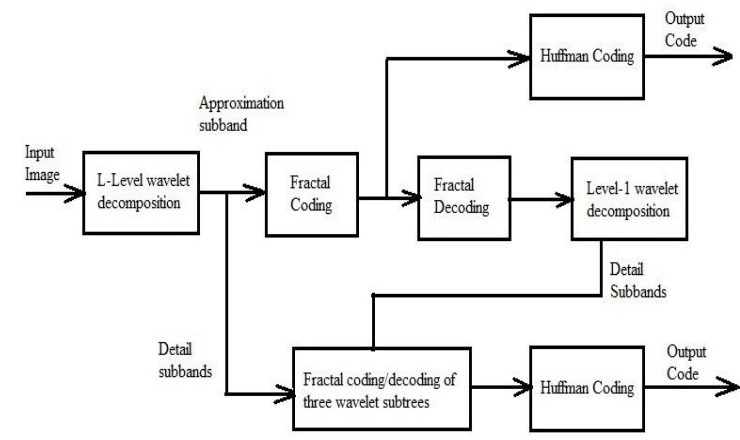

Fig 3: Proposed System

\section{COMPARATIVE ANALYSIS House OF EXPERIMENTAL RESULTS}

The results for proposed system are compared with quad tree coding for various images of size of $256 \times 256$, The compression ratio and encoding time of different color images as Girl, House, Jellybean and Tree in tiff image format are given in Table 4 . The given images are from SIPI image database.

Discrete Wavelet-based quad tree combined with Huffman coding gives more compression for level 2 and level 3 as compared with quad tree with Huffman coding but at the cost of compression time for the set of images. Fig. 4 shows graphical representation of comparison of compression ratio for different fractal techniques. The compression ratio is higher for level-3 Wavelet based Fractal Quad Tree Decomposition and Huffman Coding. The higher the level in wavelet decomposition more is the compression ratio obtained for given color images. The compression ratio obtained for level-2 and level-3 of wavelet is even greater than compression ratio of Quad tree combined with Huffman Coding. 
Table 4. Wavelet based Fractal Quad Tree Decomposition and Huffman Coding

\begin{tabular}{|c|c|c|c|c|}
\hline \multirow{2}{*}{$\begin{array}{l}\text { Methods/ } \\
\text { Images } \\
\text { parameters }\end{array}$} & \multicolumn{2}{|c|}{$\begin{array}{l}\text { Quad tree } \\
\text { combined } \\
\text { with Huffman } \\
\text { coding }\end{array}$} & \multicolumn{2}{|c|}{$\begin{array}{l}\text { Discrete wavelet based } \\
\text { quad tree combined } \\
\text { with Huffman coding }\end{array}$} \\
\hline & $\begin{array}{l}\text { Com } \\
\text { press } \\
\text { ion } \\
\text { ratio }\end{array}$ & $\begin{array}{l}\text { Encod } \\
\text { ing } \\
\text { time } \\
\text { (s) }\end{array}$ & $\begin{array}{l}\text { Compression } \\
\text { ratio }\end{array}$ & $\begin{array}{l}\text { Enco } \\
\text { ding } \\
\text { time } \\
\text { (s) }\end{array}$ \\
\hline \multirow{3}{*}{ Girl.tiff } & \multirow{3}{*}{20.14} & \multirow{3}{*}{0.99} & 6.49(level 1) & 6.13 \\
\hline & & & 27.28(level 2) & 5.62 \\
\hline & & & 102.80(level 3) & 6.42 \\
\hline \multirow{3}{*}{ House. tiff } & \multirow{3}{*}{14.97} & \multirow{3}{*}{1.21} & 6.41(level 1) & 6.41 \\
\hline & & & 27.35(level2) & 6.98 \\
\hline & & & 104.25(level3) & 5.6 \\
\hline \multirow{3}{*}{$\begin{array}{l}\text { Jelly } \\
\text { bean.tiff }\end{array}$} & \multirow{3}{*}{22.88} & \multirow{3}{*}{0.94} & 6.72(level 1) & 6.72 \\
\hline & & & 28.36(level 2) & 6.11 \\
\hline & & & 114.13(level3) & 8.08 \\
\hline \multirow{3}{*}{ Tree.tiff } & \multirow{3}{*}{8.57} & \multirow{3}{*}{1.38} & $6.25($ level 1) & 8.95 \\
\hline & & & 25.67(level 2) & 5.58 \\
\hline & & & 88.65(level3) & 8.26 \\
\hline
\end{tabular}

Comparison of Compression Ratio by diffrent Fractal Techniques

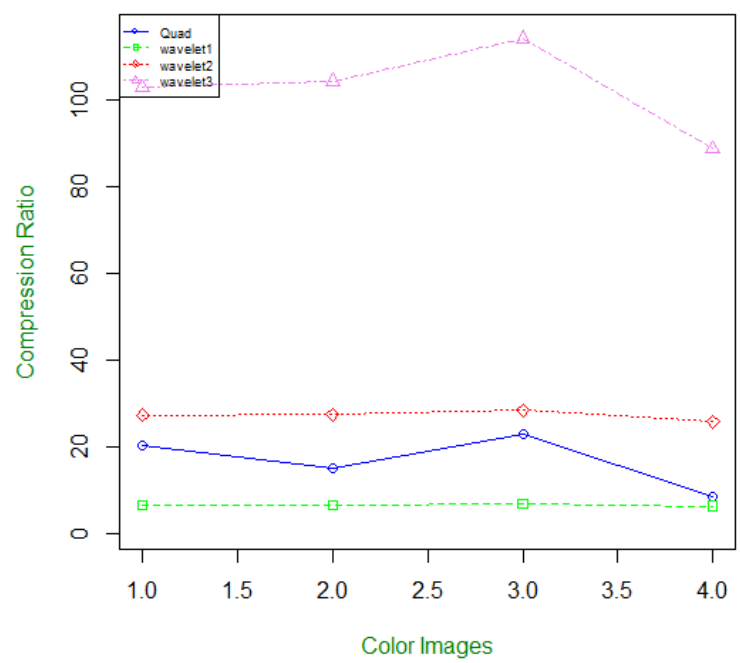

Fig 4: Comparison of Compression ratio by different fractal techniques.

\section{CONCLUSION AND SCOPE FOR RESEARCH}

Fractal image compression methods take longer encoding time. It is a lossy compression method. Fuzzy and ANN techniques are used to classify domain and range blocks. Also, different genetic and search algorithms are used to reduce the encoding time. In this paper, comparison of a Lena image, texture image and satellite image is given for existing fractal based methods. Wavelet based fractal Quad tree system is implemented and results are obtained. Also, the analysis and comparison of different parameters as encoding time, decoding time, compression ratio, PSNR etc. is done for each of the existing fractal coding techniques. The discrete wavelet transform with fractal image compression using quad tree decomposition with Huffman encoding is used for achieving better image compression which is compared with Quad tree combined with Huffman coding, but at the cost of encoding time.

Further research can be carried out to reduce the encoding time. The concept of wavelet can be combined with fractal video coding as an extension to this work. Also, analysis of proposed system can be done using medical images which are hard to compress.

\section{REFERENCES}

[1] Y. Fisher, Fractal image compression: Theory and applications, 1995.

[2] Feng-qing Qin, Jun Min, Hong-rong Guo, De-hui Yin, "A Fractal Image Compression Method based on block classification and quad tree partition", IEEE computer society, pp 716-719, 2009.

[3] Jianji Wang, "A Novel Fractal Image Compression Scheme with Block Classification and Sorting Based on Pearson's Correlation Coefficient ", IEEE TRANS. ON IMAGE PROCESSING, pp 3690-3702, vol. 22, No. 9, September 2013.

[4] A.Muruganandhama, Dr. R.S.D. Wahida B, "Adaptive Fractal Image Compression using PSO", Procedia Computer Science 2 (2010), 338-344, ICEBT, 2010.

[5] Sridharan Bhavani, Kepanna Gowder Thanushkodi, "Comparison of fractal coding methods for medical image compression", IET Image Process., 2013, Vol. 7, Iss. 7, pp. 686-693.

[6] Tama's Kova'cs, "A fast classification based method for fractal image encoding", Elsevier, Vision Computing, 26 (2008) 1129-1136.

[7] Hsiu-Niang Chen , Kuo-Liang Chung, Jian-Er Hung, "Novel fractal image encoding algorithm using normalized one-norm and kick-out condition", Image and Vision Computing, 28 (2010) 518-525.

[8] Zhang Chao,"Fast Fractal Image Encoding Based on Special Image Features", TSINGHUA SCIENCE AND TECHNOLOGY pp58-62 Volume 12, Number 1, February 2007.

[9] Zhou Y, Zhang Chao and Zhang Z,"Fast Fractal Image Encoding Using an Improved Search Scheme", TSINGHUA SCIENCE AND TECHNOLOGY, pp602606 Volume 12, Number 5, October 2007. 
[10] K. Jaferzadeh K, Kiani S. Mozaffari, "Acceleration of fractal image compression using fuzzy clustering and discrete-cosine-transform-based metric", IET, 2012.

[11] SONG Chun-lin, FENG Rui, LIU Fu-qiang1, CHEN Xi, "A Novel Fractal Wavelet Image Compression Approach", Journal of China University of Mining \& Technology, Vol.17 No.1, March 2007.

[12] Jyh-Horng, Chun-Chieh T and Jer-Guang Hsieh, "Study on Huber Fractal Compression," IEEE TRANSACTIONS ON IMAGE PROCESSING, VOL. 18, NO. 5, pp.995-1003, MAY 2009.

[13] Yuzo Iano, F Silvestre da Silva, and Ana Lúcia Mendes Cruz, "A Fast and Efficient Hybrid Fractal-Wavelet Image Coder", IEEE TRANSACTIONS ON IMAGE PROCESSING, vol. 15, issue 1, January 2006.

[14]Yi-Ming Zhou, Chao Zhang, Zeng ke Zhang, "Fast hybrid image compression using an image feature and neural network", Chao's, Solitons and Fractals, vol 37, issue 2, 2008

[15] Songlin Du, Yaping Yan, and Yide M, "QuantumAccelerated Fractal Image Compression: An Interdisciplinary Approach", IEEE SIGNAL PROCESSING LETTERS, VOL. 22, NO. 4, 499-503, APRIL 2015.

[16] Ching Hung Yuen , Oi Yan Lui, Kwok Wo Wong, “ Hybrid fractal image coding with quadtree-based progressive structure", J. Vis. Commun. Image R. 24 (2013) 1328-1341.

[17] Ming-Sheng Wu, "Genetic algorithm based on discrete wavelet transformation for fractal image compression" ,J. Vis. Commun. Image R. vol 25,issue 8, (2014) 18351841.

[18] Jianji Wangn, NanningZheng, YuehuLiu,GangZhou, "Parameter analysis of fractal image compression and its applications in image sharpening and smoothing", Signal Processing: Image Communication, 28 (2013)681-687.

[19] Yi Zhang, Xingyuan Wang, "Fractal compression coding based on wavelet transform with diamond search", Nonlinear Analysis: Real World Applications, 13 (2012) 106-112.

[20] Wang Xing-yuan, Li Fan-ping, Wang Shu-Guo, "Fractal Image Compression based on spatial correlation and hybrid genetic algorithm", Journal of Vis. Comm. and Image Representation, Vol 20, Issue 8, pp. 505-510, 2010 .

[21] Shiping Zhu, Liyun Li, Jugiang Chen, Kamel Belloulata, "An automatic region- based video sequence codec based on fractal compression", vol.68, issue 8,pp.795-805, August 2014.

[22] Sandhya Kadam, Vijay Rathod, "Analysis of color and medical images using H.264/AVC”, PNFE, 2016.

[23] Hamid Salimi, "Stochastic Fractal search: A powerful metaheuristic algorithm", Knowledge-based systems, vol.75, pp.1-18, February 2015.

[24] Wavelets and fractals in earth system sciences- V M Gadre

[25] A wavelet tour of signal processing, Stephane Mallat.
[26] Bohong Liu, Ying Yan, "An improved fractal image coding based on the quadtree", 3rd IEEE Int.Congress on Image and Signal Processing, vol. 2, pp. $529-532$, 2010.

[27] Kamran M, Irshad Sipra A, Nadeem, M, "A novel domain optimization technique in fractal image compression", IEEE Conf. on Intelligent Control -and Automation (WCICA), pp. 994- 999, 2010.

[28] Cangju Xing, “An Adaptive Domain Pool Scheme for Fractal Image Compression", IEEE International Workshop on Education Technology and Training and Geoscience and Remote Sensing, vol.2, pp.719-722, 2008.

[29] Chong Fu, Zhi-liang Zhu, "A DCT-Based Fractal Image Compression Method", International Workshop on Chaos- Fractals Theories and Applications, pp. 439443,2009

[30] Sandhya Kadam, Vijay Rathod, "DCT with Quad tree and Huffman Coding for color images ", International Journal of Computer Applications, vol. 173, no.9, pp. 3337, 2017.

[31] Kiani, K, Jaferzadeh K, Rezaie H, Gholami S, "A New Simple Fast DCT Coefficients-Based Metric Operation for Fractal Image Compression", IEEE Second International Conference on Computer Engineering and Applications (ICCEA), pp. 51-55, 2010

[32] Salarian, M, Hassanpour H, "A new fast no search fractal image compression in DCT domain", International Conference on Machine Vision, pp.62-66, 2007.

[33] Jinshu Han “ Fast Fractal Image Compression Using Fuzzy Classification", International Conference on Fuzzy Systems and Knowledge Discovery, 2008.

[34] Kung, C.M, Yang, W.S, Ku, C.C, Wang, C.Y , "Fast Fractal Image Compression Base on Block Property", Int. Conf. on Advanced Computer Theory and Engineering, pp. $477-481,2008$.

[35] Prasad, V.R., Vaddella, Babu, R, Inampudi, “Adaptive Gray Level Difference to Speed Up Fractal Image Compression", IEEE Int. Conf. on Signal Processing, Communications and Networking, pp. 253 - 258 , 2007

[36] George, L.E. Al-Hilo, E.A. "Isometric process behavior in fractal color image compression by zero- mean method", Int. Con. on Computer Engineering and Technology, 2010

[37] George, L.E, Al-Hilo, E.A., "Fractal Color Image Compression by Adaptive Zero-Mean Method", International Conference on Computer Technology and Development, vol.1, pp.525-529, 2009.

[38] Al-Hilo, E.A, George, L.E., "Speeding up fractal colored image Compression”, IEEE Conf. on Digital

Image Computing: Techniques and Applications, pp. 486 - 490, 2008.

[39] Selim, A, Hadhoud, M.M, Dessouky, M.I, Abd El-Samie, F.E, "A simplified fractal image compression algorithm", IEEE International Conference on Computer Engineering \& Systems, pp. 53-58, 2008. 
[40] Zhuang Wu, Bixi Yan, "An effective fractal image compression algorithm", International Conference on Computer application and system Modelling, 2010.

[41] R. E. Chaudhari, S. B. Dhok, "Wavelet Transform based Fast Fractal Compression", International Conference on Circuits, Systems, Communication and Information Technology Applications, 2014.

[42] Sandhya Kadam, Vijay Rathod, " Fractal coding for Texture, Satellite and gray scale images to reduce searching time and complexity", FICTA, 2017.

[43] Vidhya, K, Shenbagadevi, S, "Performance Analysis of Medical Image Compression", IEEE International
Conference on Signal Processing Systems, pp. 979 - 983, 2009.

[44] Jinshu Han," Fast Fractal Image Encoding based on Local variances and genetic algorithm", IEEE Conference, 2009.

[45] Bobde, Sarika Sanjay; Kulkarni, M.V, Kulkarni, P.V, "Fractal Image Compression Using Genetic Algorithm", International Conference on Advances in Computer Engineering (ACE), pp. 241 - 243, 2010.

[46] Sulema, Y.; Kahou, S.E., "Image compression: Comparative analysis of basic algorithms ", IEEE Conf. on Design \& Test Symposium (EWDTS), pp.534 - 537, 2010. 